EESTI NSV TEADUSTE AKADEEMIA TOIMETISED. XI KOIDE

FOOSIKALIS-MATEMAATILISTE JA TEHNILISTE TEADUSTE SEERIA. 1962, NR. 2

ИЗВЕСТИЯ АКАДЕМИИ НАУК ЭСТОНСКОИ ССР. ТОМ ХІ

СЕРИЯ ФИЗИКО-МАТЕМАТИЧЕСКИХ И ТЕХНИЧЕСКИХ НАУҚ. 1962, Ni 2

\title{
МЕТОД РЕШЕНИЯ ОБОБЩЕННОЙ ТРАНСПОРТНОЙ ЗАДАЧИ
}

\author{
и. кулль, \\ кандидат физико-математических наук
}

Э. ЛАНДРА

На Четвертом всесоюзном математическом съезде в июле 1961 г. в докладах В. В. Шкурбы и М. К. Гавурина, Г. Ш. Рубинштейна и С. С. Сурина (['], стр. 96) были изложены методы решения обобщенной транспортной задачи, основанные на методе потенциалов. Ниже дается более простой метод решения, особенно удобен для нахождения почти оптимальных планов (см. табл. 5), основанный на методе А. Лурье $\left[{ }^{2}\right]$.

1. С обобщенными транспортными задачами мы имеем дело, например, при распределении различных видов топлива между различными энергетическими установками. Так как фактическое содержание величин не суживает задачи, можем при формулировке задачи прнменить термины энергетической проблемы.

Пусть даны $m$ видов топлива с запасами соответственно $a_{1}, a_{2}, \ldots, a_{m}$ (напри мер, в ккал). Из них некоторые виды топлива (скажем, $\kappa$ первых) являются прннудительнымн, подлежащими обязательному израсходованию. Кроме того, даны $n$ энергетических установок, с количествами производимого тепла соответственно $b_{1}$, $b_{2}, \ldots, b_{n}$ (в ккал). Предполагаем, что общий топливный резерв позволяет этн установки нагрузить полностью. Даны еще коэффнциенты $c_{i j} \geqslant 0 \quad(i=1,2, \ldots, m$; $j=1,2, \ldots, n)$, означающие затраты на единицу производимого тепла $j$-той установкн при $i$-том внде топлива, и $\eta_{i j}>0(i=1,2, \ldots, m ; j=1,2, \ldots, n)$ - коэффицненты полезного действия $j$-той установки при $i$-том виде топлива.

Задача состонт в том, чтобы найти величнны $x_{i j} \geqslant 0(i=1,2, \ldots, m ; j=1$, $2, \ldots, n)$ - количества производимого тепла $j$-той установки при $i$-том виде топлива. прн которых общая затрата на производство тепла

$$
\sum_{i=1}^{m} \sum_{j=1}^{n} c_{i j} x_{i i}
$$

была бы миннмальной и выполнялись бы следующие условия:

$$
\sum_{i=1}^{m} x_{i j}=b_{j} \quad(j=1,2, \ldots, n)
$$

- все установки полностью нагружены, и 


$$
\sum_{j=1}^{n} \frac{x_{i j}}{\eta_{i j}} \leqslant a_{i} \quad(i=1,2, \ldots, m)
$$

- расходование топлива лимитируется соответствующими запасами $\left(\frac{x_{i j}}{\eta_{i j}}=y_{i j}\right.$ означает расходы $i$-того вида топлива для $j$-той установки). Для видов прннудительного топлива должно выполняться равенство

$$
\sum_{j=1}^{n} \frac{x_{i j}}{\eta_{i j}}=a_{i} \quad(i=1,2, \ldots, k ; k<m) .
$$

Чтобы обобщить метод А. Лурье, нужно принять в расчет и остатки видов топ лива. Эти остатки обозначаем соответственно $x_{1, n+1}, x_{2, n+1}, \ldots, x_{m, n+1}$. В оконча тельном решении все эти остатки должны быть неотрицательными. Нужно задать и коэффициенты $c_{i, n+1}$. Для принудительного топлива принимаем $c_{i, n+1}$ равным некоторому очень большому числу, для остальных видов топлива принимаем $c_{i, n+1}=0$. Коэффициенты $\eta_{i, n+1}$ принимаем все равными единице.

Тогда задача сводится к нахождению величнн $x_{i j} \geqslant 0 \quad(i=1,2, \ldots, m$; $j=1,2, \ldots, n, n+1)$, минимизирующих линейную форму

$$
\sum_{i=1}^{m} \sum_{j=1}^{n+1} c_{i j} x_{i j}
$$

и удовлетворяющих условиям (2) и

$$
\sum_{j=1}^{n+1} \frac{x_{i j}}{\eta_{i j}}=a_{i} \quad(i=1,2, \ldots, m)
$$

\begin{tabular}{|c|c|c|c|c|c|}
\hline & $b_{1}$ & $b_{2}$ & $\cdots$ & $b_{n}$ & столбец остатков \\
\hline$a_{1}$ & $c_{11} \eta_{11} \begin{array}{l}x_{11} \\
y_{11}\end{array}$ & $\begin{array}{lll} & & x_{12} \\
c_{12} & \eta_{12} & y_{12}\end{array}$ & & $\begin{array}{ccc} & & x_{1 n} \\
c_{1 n} \eta_{1 n} & y_{1 n}\end{array}$ & $c_{1, n+1} \quad 1 \quad x_{1, n+1}$ \\
\hline$a_{2}$ & $c_{21} \eta_{21} \begin{array}{l}x_{21} \\
y_{21}\end{array}$ & $\begin{array}{ll}c_{22} \eta_{22} & x_{22} \\
y_{22}\end{array}$ & $\cdots$ & $c_{2 n} \eta_{2 n} \begin{array}{l}x_{2 n} \\
y_{2 n}\end{array}$ & $c_{2, n+1} \quad 1 \quad x_{2, n+1}$ \\
\hline$\vdots$ & $\cdots$ & $\cdots$ & $\cdots$ & $\cdots$ & $\cdots$ \\
\hline$a_{m}$ & $c_{m 1} \eta_{m 1} \begin{array}{l}x_{m 1} \\
y_{m 1}\end{array}$ & $c_{m 2} \eta_{m 2} \begin{array}{c}x_{m 2} \\
y_{m 2}\end{array}$ & & $c_{m n} \eta_{m n} x_{m n}$ & $c_{m, n+1} 1 x_{m, n+1}$ \\
\hline
\end{tabular}

Набор таких величин $x_{i j}$ назогем оптимальным планом. Все начальные данные н элементы решения $x_{i j}, y_{i j}$ можно свести в одну таблицу:

Так как в столбце остатков $x_{i, n+1}=y_{i, n+1}$, то последние числа не выписываются вторично.

2. Метод решения этой задачи базируется на том, что если имеется оптимальныі план $x_{i j}$ в случае коэффициентов $c_{i j}+\frac{\lambda_{i}}{\eta_{i j}}$, где $\lambda_{i}-$ произвольные вещественные числа для строк. то этот план является оптнмальным н в случае первоначальных коэффициентов $c_{i j}$. 
В самом деле, пусть $x_{i j}$ - оптимальный план в случае коэффнцнентов $c_{i j}+\frac{\lambda_{i}}{\eta_{i j}}$ и $x_{i j}^{\prime}-$ любой другой план, удовлетворяющий условиям (2) и (6). Тогда по предположению

$$
\sum_{i=1}^{m} \sum_{j=1}^{n+1}\left(c_{i j}+\frac{\lambda_{i}}{\eta_{i j}}\right) x_{i j} \leqslant \sum_{i=1}^{m} \sum_{j=1}^{n+1}\left(c_{i j}+\frac{\lambda_{i}}{\eta_{i j}}\right) x^{\prime}{ }_{i j}
$$

Раскрывая скобки, получаем

$$
\sum_{i=1}^{m} \sum_{j=1}^{n+1} c_{i j} x_{i j}+\sum_{i=1}^{m} \lambda_{i} \sum_{j=1}^{n+1} \frac{x_{i j}}{\eta_{i j}} \leqslant \sum_{i=1}^{m} \sum_{j=1}^{n+1} c_{i j} x_{i j}^{\prime}+\sum_{i=1}^{m} \lambda_{i} \sum_{j=1}^{n+1} \frac{x_{i j}^{\prime}}{\eta_{i j}} .
$$

Учитывая условие (6), получаем равенство

$$
\sum_{i=1}^{m} \lambda_{i} \sum_{j=1}^{n+1} \frac{x_{i j}}{\eta_{i j}}=\sum_{i=1}^{m} \lambda_{i} \sum_{j=1}^{n+1} \frac{x_{i j}^{\prime}}{\eta_{i j}}=\sum_{i=1}^{m} \lambda_{i} \alpha_{i}
$$

на основанин которого из (7) вытекает

$$
\sum_{i=1}^{m} \sum_{j=1}^{n+1} c_{i j} x_{i j} \leqslant \sum_{i=1}^{m} \sum_{j=1}^{n+1} c_{i j} x_{i j}^{\prime}
$$

т. е. план $x_{i j}$ является оптимальным и в случае коэффициентов $c_{i j}$.

Оптимальный план вычисляется по нашему методу аналогично методу Лурье и Лурье-Олейннка [3]. Во-первых, прикрепляется всем установкам топливо с самыми меньшими $c_{i j}$, не учитывая вообще запасы топлива. После этого вычисляются остатки топлива. Метод требует приписания определенного знака и остаткам 0. Это определяется так: если найдется строка с отрицательным остатком, связанная с данной строкой, и эта последняя не связана ни с одной строкой с положительным остатком, то данная строка имеет остаток -0 . Если для строки с остатком 0 не найдется ни одной связанной с ней строки с отрицательным остатком, то данная строка имеет остаток +0 .

Если найдутся строки с отрнцательными остатками, связанные с данной строкой (нмеющей остаток 0), и эта последняя связана 'о строками с положительными остатками, то при помощи перенесения некоторых величин $x_{i j}$ можно этот случай свести к предыдущим нли остаток 0 не сохраняется.

Строка с номером $\mu$ считается связанной с $v$-той строкой, если имеется индекс $j$, прн котором

$$
c_{\mu, j}=c_{\nu j} \quad \text { н } \quad x_{\mu j}>0,
$$

т. е., если возможно без увеличения затрат перенести количество $x_{\mu j}$ из $\mu$-той строки в $v$-тую строку.

Перенесение количеств $x_{i j}$ из строк $\{\mu\}$ с отрицательными остатками в строки $\{v\}$ с положительными остатками производится тогда следующим образом: нужно найти минимальное (по всем $\mu, v$ и $j$ ) $\lambda$, при котором

$$
c_{1 \mu j}+\frac{\lambda}{\eta_{p j}}=c_{v j}
$$

гле $\boldsymbol{x}_{i \mu j}>0$. Таким образом, 


$$
\lambda=\min _{\mu, \nu, j}\left(c_{\nu j}-c_{\mu j j}\right) \eta_{\mu, j}
$$

где $\mu$ - индекс строк с отрнцательным остатком, $v$ - нндекс строк с положительным остатком н $x_{\mu j}>0$.

После нахождения $\lambda$ нужно вычислить новые коэффициенты для $\mu$-той строки.

$$
c_{\mu j}^{\prime}=c_{\mu j}+\frac{\lambda}{\eta_{\mu j}} \text {. }
$$

Эти преобразования должны продолжаться до тех пор, пока не будет найден олтимальный план.

Отметим, однако, что не всегда легко судить, получен ли оптимальный план. Например, если все $x_{i j}$ неотрицательны и их распределение по всем столбцам оптимальное (см. таблицу 5), то это еще не гарантирует оптимальности всего плана. Именно, может существовать план $x_{i j}^{\prime}$, имеющий неотрицательные компоненты при тех же индексах $i$, $j$, что и в плане $x_{i j}$, и меньше остатки, чем в плане $x_{i j}$. Переход от плана $x_{i j}$ к плану $x_{i j}^{\prime}$ уменьшает значение линейной формы на величину

$$
\sum_{i=1}^{m} c_{i, n+1}^{\prime} x_{i, n+1}-\sum_{i=1}^{m} c_{i, n+1}^{\prime} x_{i, n+1}^{\prime} .
$$

Отсюда получаем вывод, что значение линейной формы при оптимальном распределенин $x_{i j}$ по всем столбцам отличается от значеннй линейной формы оптимального плана не больше чем на величину

$$
\sum_{i=1}^{m} c_{i, n+1}^{\prime} x_{i, n+1}
$$

н. если эта последняя равна нулю, то получен оптимальный план. Из этого вытекает, что после оптимального распределения $x_{i j}$ по всем столбцам нужно при помощи преобразований (9) и перераспределений $x_{i j}$ минимизировать величину (10).

\section{3. Пример.}

\begin{tabular}{|c|c|c|c|c|c|c|c|c|c|}
\hline & & \multicolumn{2}{|c|}{$b_{1}=15$} & \multicolumn{2}{|c|}{$b_{2}=20$} & $b_{3}=25$ & $b_{4}=30$ & \multicolumn{2}{|c|}{ столбец остатков } \\
\hline$a_{1}=$ & 40 & 8 & 0,7 & 7 & 0,8 & 70,6 & $9 \quad 0,5$ & 1000 & 1 \\
\hline$a_{2}=$ & 50 & 4 & 0,6 & 3 & 0,7 & $4 \quad 0,5$ & 80,8 & 0 & 1 \\
\hline$a_{3}=$ & 50 & 3 & 0,5 & 5 & 0,8 & $\begin{array}{ll}6 & 0,6\end{array}$ & $\begin{array}{ll}5 & 0.7\end{array}$ & 0 & 1 \\
\hline$a_{4}=1$ & 000 & 6 & 0,6 & & 0.7 & 50,5 & $\begin{array}{ll}7 & 0,8\end{array}$ & 0 & 1 \\
\hline
\end{tabular}

Найдем решение обобщенной транспортной задачи, начальные данные которой приведены в следующей таблице:

Таблища 1 
Из таблицы видно, что первое топливо является принудительным. Прикреплял установкам самое дешевое топливо, получаем:

Таблица 2

\begin{tabular}{|c|c|c|c|c|c|c|c|c|c|c|c|c|c|c|}
\hline & & \multicolumn{3}{|c|}{$b_{1}=15$} & \multicolumn{2}{|c|}{$b_{2}=20$} & \multicolumn{3}{|c|}{$b_{3}=25$} & \multicolumn{2}{|c|}{$b_{4}=30$} & \multicolumn{3}{|c|}{ столбец остатков } \\
\hline$a_{1}=$ & 40 & 8 & 0,7 & & 7 & 0,8 & 7 & 0,6 & & 9 & 0,5 & 1000 & 1 & +40 \\
\hline$a_{2}=$ & 50 & 4 & 0,6 & & & $0,7 \stackrel{20}{28,6}$ & 4 & 0,5 & $\begin{array}{l}25 \\
50 \\
\end{array}$ & 8 & $-0,8$ & 0 & 1 & $-28,6$ \\
\hline$a_{3}=$ & 50 & 3 & 0,5 & $\begin{array}{l}15 \\
30\end{array}$ & 5 & 0,8 & 6 & 0,6 & & 5 & $0,7^{30} 42.9$ & 0 & 1 & $-22,9$ \\
\hline$a_{4}=$ & 000 & 6 & 0,6 & . & 6 & 0,7 & 5 & 0,5 & & & 0,8 & 0 & 1 & +1000 \\
\hline
\end{tabular}

Вычисляем величнну $\lambda$. По столбцам найдем

$$
\begin{array}{ll}
(6-3) \cdot 0,5=1,5 ; & (6-3) \cdot 0,7=2,1 ; \\
(5-4) \cdot 0,5=0,5 ; & (7-5) \cdot 0,7=1,4 .
\end{array}
$$

Значит, $\lambda=0,5$ и к коэффициентам второй строки нужно прибавить соответственно величины $\frac{0,5}{0,6}=0,8, \frac{0,5}{0,7}=0,7, \frac{0,5}{0,5}=1, \frac{0,5}{0,8}=0,6$ и $\frac{0,5}{1}=0,5$. Коэффициентам третьей строки нужно прибавить соответственно величины $\frac{0,5}{0,5}=1,0 ; \frac{0,5}{0,8}=0,6$; $\frac{0,5}{0,6}=0,8 ; \frac{0,5}{0,7}=0,7$ и $\frac{0,5}{1}=0,5$. После сложения и частичного перенесения $x_{23}$ получаем следующее распределение:

\begin{tabular}{|c|c|c|c|c|c|c|c|c|c|c|c|c|c|}
\hline & & \multicolumn{3}{|c|}{$b_{1}=15$} & \multicolumn{2}{|c|}{$b_{2}=20$} & \multicolumn{2}{|c|}{$b_{3}=25$} & \multicolumn{2}{|c|}{$b_{4}=30$} & \multicolumn{2}{|c|}{ столбец } & остатков \\
\hline$a_{1}=$ & 40 & 8 & 0,7 & & 7 & 0,8 & 7 & 0,6 & 9 & 0,5 & 1000 & 1 & +40 \\
\hline$a_{2}=$ & 50 & 4,8 & 0,6 & & 3,7 & $0,7 \begin{array}{l}20 \\
28,6\end{array}$ & 5 & $0,5 \begin{array}{l}10,7 \\
21,4\end{array}$ & 8,6 & 0,8 & 0,5 & 1 & + \\
\hline$a_{3}=$ & 50 & 4 & 0,5 & & 5,6 & 0,8 & 6,8 & 0,6 & 5,7 & $0,7 \begin{array}{l}30 \\
42,9 \\
\end{array}$ & 0,5 & 1 & $-22,9$ \\
\hline$a_{4}=$ & 000 & 6 & 0,6 & & 6 & 0,7 & 5 & $0,5 \begin{array}{r}14,3 \\
28,6\end{array}$ & 7 & 0,8 & 0 & 1 & $+971,4$ \\
\hline
\end{tabular}

Таблица 3

Так как имеются отрнцательные остатки, нужно вычисления продолжить. Вычисляем $\lambda ;(4,8-4) \cdot 0,5=0,4 ;(7-5,7) \cdot 0,7=0,9$. Значит $\lambda=0,4$ и к коэффнциентам третьей строки нужно прибавить величнны 0,$8 ; 0,5 ; 0,7 ; 0,6$ и 0,4 , соответственно. Частично перенеся $x_{31}$ и $x_{23}$, получаем: 
Таблица 4

\begin{tabular}{|c|c|c|c|c|c|c|c|c|c|c|c|}
\hline \multirow[b]{2}{*}{$a_{1}=$} & \multirow[b]{2}{*}{40} & \multicolumn{2}{|c|}{$b_{1}=15$} & \multicolumn{2}{|c|}{$b_{2}=20$} & \multicolumn{2}{|c|}{$b_{3}=25$} & \multicolumn{2}{|c|}{$b_{4}=30$} & \multicolumn{2}{|c|}{ столбец остатков } \\
\hline & & 8 & 0,7 & 7 & 0,8 & 7 & 0,6 & 9 & 0,5 & $1000 \quad 1$ & +40 \\
\hline$a_{2}=$ & 50 & 4,8 & $0,6{ }^{11,45}$ & 3,7 & $0,7 \begin{array}{l}20 \\
28,6\end{array}$ & 5 & $0,5 \begin{array}{c}1,15 \\
2,3 \\
\end{array}$ & 8,6 & 0,8 & $0,5 \quad 1$ & + \\
\hline$a_{3}=$ & 50 & 4,8 & $0,5 \begin{array}{c}3,55 \\
7.1 \\
\end{array}$ & 6,1 & 0,8 & 7,5 & 0,6 & 6,3 & $0,7 \begin{array}{l}30 \\
42,9 \\
\end{array}$ & $0,9 \quad 1$ & + \\
\hline$a_{4}=1$ & 000 & 6 & 0,6 & 6 & 0,7 & 5 & $0,5^{23,85}$ & 7 & 0,8 & 0 & $+952,3$ \\
\hline
\end{tabular}

Так как полученное распределение остатков явно неоптимальное, то нужно уменьшить коэффициенты $c_{1 j}$ первой строки. Вычисляем $\lambda ;(8-4,8) \cdot 0,7=2,24 ;(7-3,7) \cdot 0,8=$ $=2,64 ;(7-5) \cdot 0,6=1,2 ;(9-6,3) \cdot 0,5=1,35$. Значит, $\lambda=-1,2$ и соответствующие слагаемые будут $-1,7 ;-1,5 ;-2 ;-2,4 ;-1,2$. Перенеся $x_{43}$ и (частично) $x_{23}$, получаем:

Таблица 5

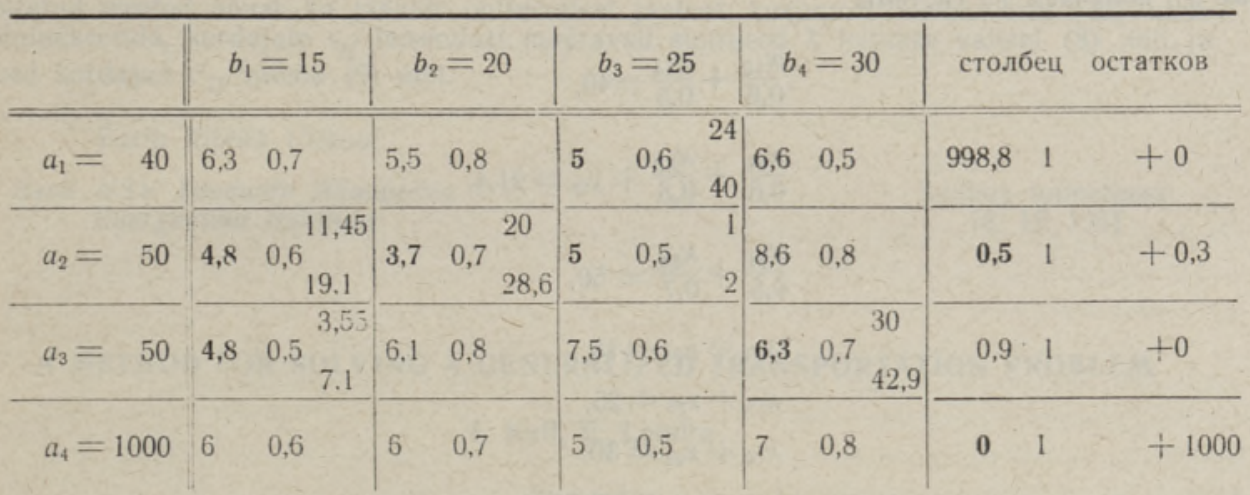

Полученное распределение величнн $x_{i j}$ по всем столбцам уже оптимальное (коэффицненты $c_{4 j}$ последней строки можно увеличить при помощи $\left.\lambda=0,5\right)$. Общие затрать: (1) на основе первоначальных коэффициентов при данном плане равны 438,5 ед. Однако мы не можем утверждать, что получен оптимальный план. На основе таблицы 5 и формулы (10) можем лишь сказать, что значение линейной формы при данном плане превышает наименьшее значение не более чем на 0,15 ед.

Исходя из таблицы 5 , попробуем свести коэффициент $c_{25}$ к нулю, сохраняя при этом равенства $c_{21}=c_{31} ; c_{13}=c_{23}$ и неравенства $c_{22} \leqslant c_{12}, c_{32} ; c_{34} \leqslant c_{14}, c_{24}$. Первые три строки нужно преобразовать тогда при помощи $\lambda_{1}=-0,6 ; \lambda_{2}=-0,5$ и $\lambda_{3}=-0,4$. Но тогда получается, что $c^{\prime}{ }_{14}=5,4$ и $c^{\prime}{ }_{34}=5,7$, т. е. $c^{\prime}{ }_{14}<c^{\prime}{ }_{34}$. Последнее неравенство требовало бы перераспределения величин $x_{i 4}$, что существенно изменило бы весь план. Тогда уменьшим $c_{25}$ лишь настолько, чтобы сохранилось $c_{14} \geqslant c_{34}$. Соответствующие корректирующие $\lambda_{i}$ найдем из системы уравнений

$$
\begin{aligned}
& 0,5 \lambda_{1}=0,6 \lambda_{2}, \\
& 0,5 \lambda_{2}=0,6 \lambda_{3},
\end{aligned}
$$




$$
0,3+\frac{\lambda_{1}}{0,5}=\frac{\lambda_{3}}{0,7}
$$

получаем $\lambda_{1}=-0,29, \lambda_{2}=-0,24$ и $\lambda_{3}=-0,20$. Новые коэффициенты $c_{i j}^{\prime}$ приведены в таблище 6.

Таблица 6

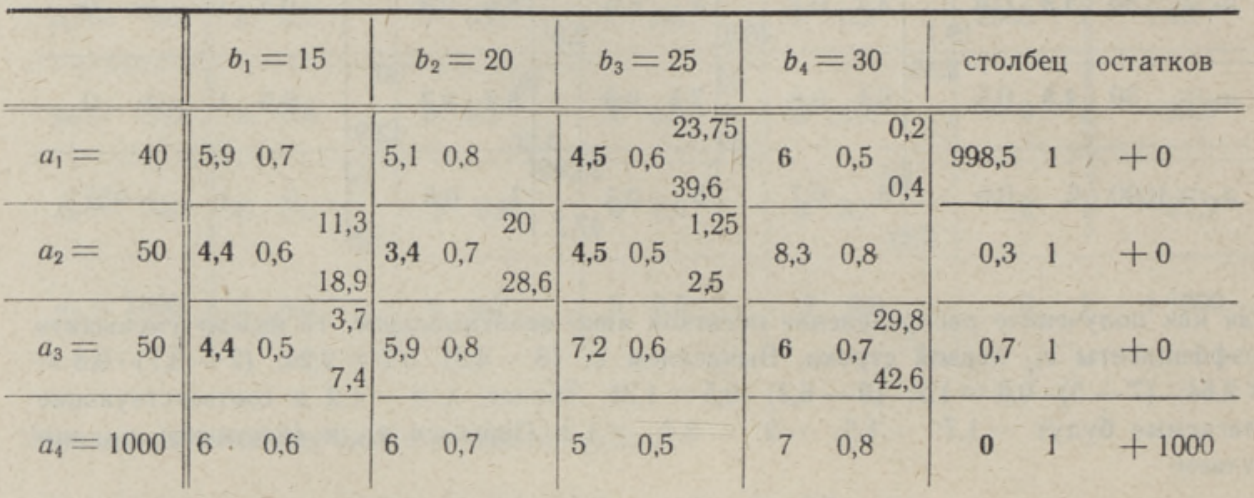

Так как $c_{25}=0,3>0$, то нужно в новом плане стремиться к минимальному $x_{25}$. Новые $x_{i j}$ получаем из системы

$$
\begin{aligned}
& \frac{x_{13}}{0,6}+\frac{x_{14}}{0,5}=40, \\
& \frac{x_{21}}{0,6}+\frac{x_{23}}{0,5}+x_{25}=21,4, \\
& \frac{x_{31}}{0,5}+\frac{x_{34}}{0,7}=50, \\
& x_{21}+x_{31}=15, \\
& x_{13}+x_{23}=25, \\
& x_{14}+x_{34}=30,
\end{aligned}
$$

учитывая требование минимальности $x_{25}$. Решение системы приведено в таблице 6 . В этом плане распределение $x_{i j}$ по всем столбцам оптимальное и, кроме того, величина (10) равна нулю. Следовательно, получен оптимальный план. Общие затраты (1) на основе первоначальных коэффициентов для этого плана равны 438,4 ед.

При практическом применении иногда удобнее использовать почти оптимальные планы, как в таблице 5 (см., напр., четвертые столбцы в таблицах 5 и 6).

\section{Тартуский государственный университет}

Ннститут энергетики Академии наук Эстонской ССР
Поступила в редакцию

18. IX 1961 


\title{
Л И Т Е Р А У Р А
}

1. Программа Четвертого всесоюзного математического съезда 3 VII 12 VII 1961 г., Ленинград 1961 г.

2. А. Л. Л ур ье, Методы достижения наименьшего пробега грузов при составлении перевозочных схем, в сб. Применение математики в экономических исследованиях, Москва 1959, стр. 354-389.

3. Ю. А. Олейник, Решение задачи о транспортировке на электронной вычнслительной машине методом приближения условно оптимальными планами, Москва 1960, 33 стр.

\section{OLDISTATUD TRANSPORDIOLESANDE LAHENDUSMEETOD}

I. Kull,

füüsikalis-matemaatiliste teaduste kandidaat

\section{E. Landra}

\section{Resümee}

Artiklis vaadeldakse üldistatud transpordiülesannet, mis esineb näiteks mitme kütuseliigi jaotamisel mitmele energeetikaseadmele. Olesanne seisneb suuruste $x_{i j} \geqslant 0$ leidmises, mis minimiseeriksid lineaarvormi (5), kusjuures oleksid täidetud tingimused (2) ja (6). Esitatud meetod on A. Lurje $\left[{ }^{2}\right]$ meetodi üldistus. Olulisem erinevus on selles, et esitatud meetod nôuab ka jääkide optimaalset jaotust, kusjuures lineaarvorm (10) tuleb minimiseerida, kordajate $c_{i j}$ teisendusi määravad suurused $\lambda$ leitakse valemi (8) abil ja uued kordajad $c^{\prime}{ }_{i j}$ valemi (9) abil.

\section{Tartu Riiklik Olikool}
Eesti NSV Teaduste Akadeemia Energeetika Instituut
Saabus toimetusse
18. IX 1961

\section{A METHOD FOR SOLVING A GENERALIZED TRANSPORTATION PROBLEM}

\author{
I. Kull, E. Landra
}

\section{Summary}

In the paper a generalized transportation problem is considered, which may occur. e. g., in the distribution of several kinds of fuel to various energy aggregates. The problem consists in the determination of the quantities: $x_{i j} \geqslant 0$, which minimize the linear form (5) and satisfy the conditions (2) and (6). The presented method is a generalization of the method of A. Lurye ${ }^{[2}$. The most important difference is that our method requires the optimal distribution of surplus fuels, and thus the linear form (10) must be minimized; the quantities $\lambda$ for the transformations of the coefficients $c_{i j}$ are determined by the formula (8), and new coefficients $c^{\prime}{ }_{i j}-$ by the formula (9).

Tartu State University

Academy of Sciences of the Estonian S.S.R., Institute of Energetics

Received Sept. 18th, 1961 